Conference Record of the 1998 IEEE International Symposium on Electrical Insulation, Arlington, Virginia, USA, June 7-10, 1998

\title{
DIGITAL RECORDING AND ANALYSIS OF PARTIAL DISCHARGES IN POINT-DIELECTRIC GAPS
}

\author{
Yicheng Wang, ${ }^{1}$ Xiaolian Han, and Richard J. Van Brunt \\ National Institute of Standards and Technology, Electricity Division, Gaithersburg, MD 20899 \\ Tadeusz Las and Helena Slowikowska \\ Electrotechnical Institute, High Voltage Department, Warsaw, Poland \\ John C. Horwath and Daniel L. Schweickart \\ Wright Laboratory, AeroPropulsion and Power Directorate, Wright Patterson AFB, OH 45433
}

\begin{abstract}
Pulsating partial discharges (PD) occurring in pointdielectric gaps under an alternating voltage have been measured with a newly developed digital recording system. The dielectric is a cast epoxy resin with $\mathrm{Al}_{2} \mathrm{O}_{3}$ filler. In this paper, we analyze and report statistical characteristics of the PD including amplitude distribution, phase-of-occurrence distribution as well as the phase distributions of the individually selected PD pulses, e. g., the first-occurring or secondoccurring pulse in each cycle.
\end{abstract}

\section{Introduction}

Partial discharges in high-voltage insulating systems may originate from various local defects such as foreign particles, voids or metallic surface intrusions. One of the goals of PD research is to relate the observable statistical characteristics of PD to properties of the defect site at which the discharge occurs and ultimately to identify the type of the defect. The success of PD identification schemes clearly requires extensive research of PD properties in a variety of well defined, artificially produced defects. Fruth and Niemeyer [1] studied ac $\mathrm{PD}$ in voids and at interfacial trees using a phase-resolved PD analyzer. They showed that each type of defect exhibits a distinct phase-resolved PD pattern. Malik and Al-rainy [2] studied statistical variations of dc PD pulse amplitudes in point-to-plane gaps in air using a multichannel analyzer. They observed that the pulse amplitudes generally follow a Gaussian-type of probability distribution. However, near the corona inception voltage or near the pulse-to-glow transition voltage level, PD pulse amplitude distributions appear to deviate from the Gaussian distribution. Van Brunt et al. [3] studied changes in the stochastic behavior of PD occurring on a dielectric surface as a function of time. They suggested that the observed changes in PD patterns can be attributed to the PD-induced increase of the surface conductivity. They also showed that the "aging effect" is accelerated as the frequency of applied voltage is increased from $50 \mathrm{~Hz}$ to 800 $\mathrm{Hz}$. In this paper, we report the dependence of PD characteristics on applied gap voltages in a small point-to-dielectric gap in air.

\section{Measurement System}

A simplified block diagram of the measurement system is shown in Fig. 1. The point-to-dielectric gap is formed in air by a $60^{\circ}$ cone-shaped stainless steel point electrode (tip radius $\sim 50 \mu \mathrm{m}$ ) and the surface of a flat cast-epoxy resin with $\mathrm{Al}_{2} \mathrm{O}_{3}$ filler $(10 \mathrm{~cm} \times 10 \mathrm{~cm})$ resting on top of a stainless steel disk plane. The PD pulse current is integrated by an integrating amplifier with a slew rate of $0.22 \mathrm{~V} / \mathrm{ns}$. The $\mathrm{RC}$ time constant of the integrator is about $20 \mu \mathrm{s}(\mathrm{C}=100 \mathrm{pF}$ and $R=200 \mathrm{k} \Omega$ ). The integrated $P D$ waveform is summed together with $2 \mu \mathrm{s}$ wide time marks from a zero-crossing detector. The summed signal is then passed to a custom

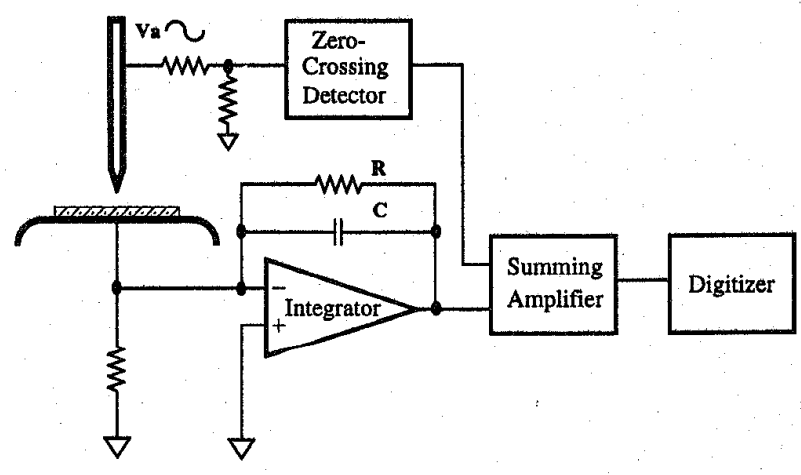

Fig. 1. Schematic diagram of the PD detection system.

\footnotetext{
${ }^{1}$ Electronic address: yicheng.wang@nist.gov
} 
digitizing PD detection system which has been described in detail by Wang [4] for $\mathrm{dc}$ applications.

The PD digitizing system uses a multifunction data acquisition board to continuously acquire the waveform which carries both $\mathrm{PD}$ and time mark information at a sample rate of 1 million samples/s with a resolution of 12 bits. The board cyclically fills a circular buffer which is configured to contain 2 million data points and is divided into two equal halves so that data acquired during one half can be transferred, analyzed, and saved while the other half is being filled. The system acquires consecutive $1 \mathrm{~s}$ PD waveforms.

\section{Results and Discussion}

The PD results presented here are obtained by applying a continuous ac voltage at $60 \mathrm{~Hz}$ to a point-to-dielectric gap of $0.5 \mathrm{~mm} \pm 0.01 \mathrm{~mm}$ in room air at a temperature of about $23{ }^{\circ} \mathrm{C}$ and a humidity of 59\%. PD for these conditions appear very stable for gap voltages slightly above the inception voltage of $1.79 \mathrm{kV}$ (measured with a $\pm 2 \%$ uncertainty $\mathrm{HV}$ probe). In general, the amplitude of the PD pulses that occur in the positive half cycle is larger than those in the negative half cycle by a factor of about 8 . The total number of positive $\mathrm{PD}$ pulses per cycle is smaller than that for negative $\mathrm{PD}$ by approximately the same factor. Figure 2 shows the normalized distributions of PD phase-of-occurrence as well as the normalized distributions of $\mathrm{PD}$ amplitude for the gap rms voltages of $1.95 \mathrm{kV}, 2.15 \mathrm{kV}, 2.40 \mathrm{kV}$, and $2.70 \mathrm{kV}$. The data at each voltage level were collected consecutively for approximately $5 \mathrm{~min}$ each, starting with a fresh dielectric surface. The threshold of the PD detection system was set at $5 \mathrm{pC}$. As can be seen in this figure, the average amplitude of positive or negative $P D$ pulses changes very little with the applied gap voltage. The number of positive PD pulses per ac cycle, however, increases consistently from one for 1.95 $\mathrm{kV}$ to four for $2.70 \mathrm{kV}$. This increase is reflected in the observable patterns of the phase distributions for positive PD. The pulse rate of negative PD also increases with the gap voltage, but the overall shape of the phase distributions for negative PD does not show any multiple-peak structure because the time separation between two adjacent negative PD pulses is much smaller than that for positive PD. Figure 3 shows the phase distribution of negative PD in Fig. 2(a) on an expanded scale together with the phase distributions for the constituent individual negative PD pulses (i.e., first occurring pulse, second occurring pulse, and so on, in the negative half cycle). The distributions of the individual pulses all show Gaussian shape, although the shape of the overall phase distribution of all negative PD pulses can not be classified with any simple mathematical formula. This Gaussian feature, which was first observed by Van Brunt $e t$ al. [3], could be utilized to characterize PD patterns in PD pattern recognition schemes.

The observed PD behavior can be better understood with the help of a simple model circuit shown in Fig. 4 where $C_{g}$ corresponds to the air gap and $C_{i}$ corresponds to the capacitance of the dielectric. The voltage across the air gap, $\mathrm{V}_{\mathrm{g}}$, in the absence of PD is linearly related to the sinusoidal voltage, $\mathrm{V}_{\mathrm{a}}$, applied to the point electrode. A discharge deposits charge on the dielectric surface resulting in a net charge transfer across the air gap. Therefore, the net charge of the lower plate of $C_{g}$ and the upper plate of $C_{j}$ shown in Fig. 4 is, in general, non-zero. Since the air gap is short and the mobilities of the typical ions in air $\left(\mathrm{N}_{2}^{+}, \mathrm{O}_{2}^{+}\right)$are large, the transit time of the ions is negligibly small compared to the period of the applied voltage. The charge transfer associated with each PD, then, causes a sharp voltage drop across the gap as illustrated in Fig. 4. If $\mathrm{V}_{\mathrm{a}}$ is sufficiently low, $\mathrm{V}_{\mathrm{g}}$ may not reach the PD inception level again within the half cycle after a $\mathrm{PD}$, as is the case for positive $\mathrm{PD}$ at $1.95 \mathrm{kV}$ [Fig. 2(a)]. The smaller amplitudes, combined with the lower inception voltage for negative PD in air, result in more negative PD pulses than positive PD. The model presented in this discussion also suggests that no positive PD should occur after $\pi / 2$ and likewise no negative PD after $3 \pi / 2$. The phase distributions shown in Fig. 2 are in general agreement with this statement; the PD pulses that occur outside the ranges represent only small fractions of the total distributions.

Although the pulse rate for positive PD is much smaller than that for negative $P D$, the average current due to positive $P D$ is remarkably close to the current due to negative $\mathrm{PD}$, as shown in Fig. 5 for $\mathrm{V}_{\mathrm{a}}=2.7 \mathrm{kV}$. The small difference between two average currents may suggest that the amplitudes of some small electron avalanche pulses that occur in the negative half cycle are below the threshold of the detection system. Figure 5 also shows that the PD currents increase with time. The increasing PD currents are associated with the increasing PD rates rather than with the PD amplitudes. The increasing PD activity is probably caused by PD-induced aging of the dielectric surface. Physical damage to the surface, however, becomes visible with scanning profilometry only after much longer exposure to PD. Figure 6 shows a profilegram of the epoxy surface after being exposed to $\mathrm{PD}$ at a $4 \mathrm{kV}, 600 \mathrm{~Hz}$ ac voltage for 26 hours.

\section{Conclusions}

While PD often exhibit non-stationary, stochastic behavior, the PD observed in a small point-to-dielectric gap appear remarkably stable. The PD phase distributions show regularly structured patterns which can be controlled through the applied gap voltage. The main features of the observed PD 

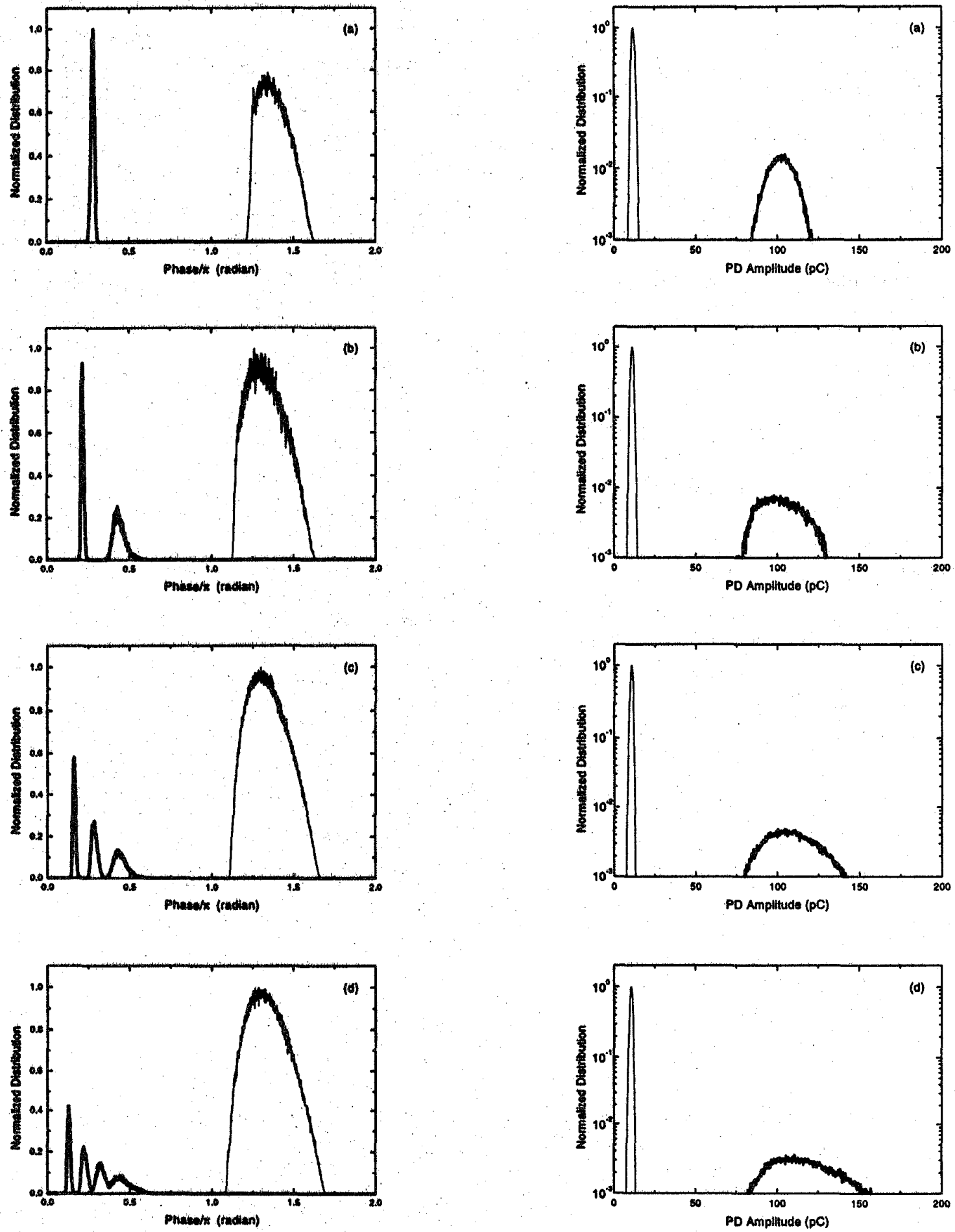

Fig. 2. PD phase-of-occurrence and amplitude distributions are normalized with the maximums equal to 1 . Thick lines are for positive PD pulses and thin lines for negative pulses. Gap voltages: (a) $1.95 \mathrm{kV}$, (b) $2.15 \mathrm{kV}$, (c) $2.40 \mathrm{kV}$, (d) $2.70 \mathrm{kV}$. 


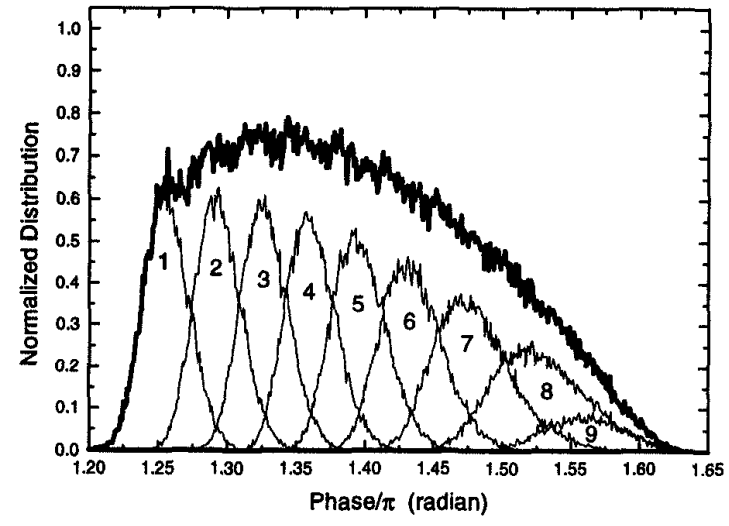

Fig. 3. Measured phase distributions for individual (thin line) and all (thick line) negative PD pulses at $1.95 \mathrm{kV}$. Numbers represent pulse sequence within negative phase.

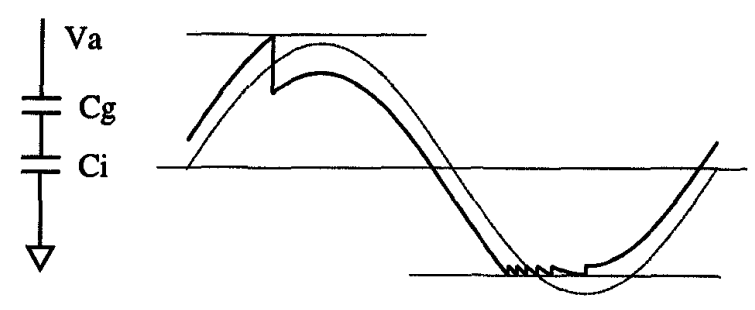

Fig. 4. Real voltage (solid line) across the gap deviates from the applied voltage (dashed line) because of the surface charge produced by PD.

can be explained with a simple two-capacitor model. More work on this type of regular, understandable PD patterns will undoubtedly help improve and facilitate the use of pattern recognition for diagnosing HV apparatus.

\section{References}

[1]. B. Fruth and L. Niemeyer, "The importance of statistical characteristics of partial discharge data," IEEE Trans. Electr. Insul. 27, 60 (1992).

[2]. N.H. Malik and A.A. Al-rainy, "Statistical variations of de corona pulse amplitudes in point-to-plane air gaps," IEEE Trans. Electr. Insul. 22, 825 (1987).

[3]. R.J. Van Brunt, P. von Glahn, and T. Las, "Anomalous stochastic behavior of partial discharge on aluminum oxide surfaces," J. Appl. Phys. 81, 840 (1997).

[4]. Y. Wang, "New method for measuring statistical distributions of partial discharge pulses," J. Res. Natl. Inst. Stand. Technol. 102, 569 (1997).
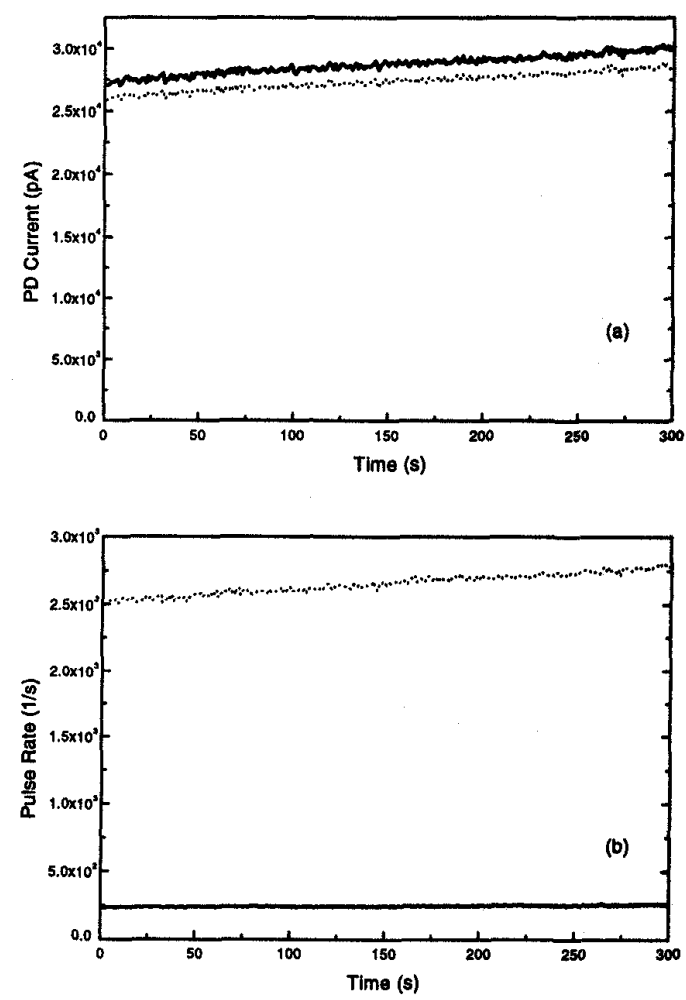

Fig. 5. (a) Integrated current. (b) Pulse rate. Solid lines are for positive $\mathrm{PD}$ pulses and dotted lines for negative pulses. Gap voltage: $2.70 \mathrm{kV}$.

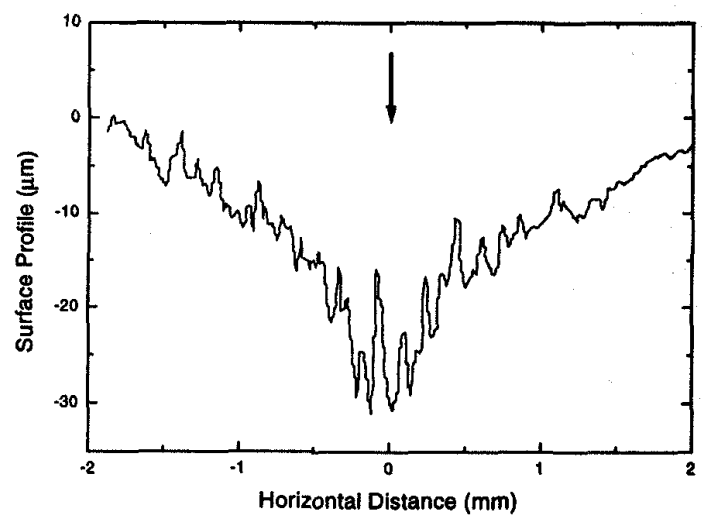

Fig. 6. Profilegram of the epoxy surface after exposure to PD. The vertical arrow indicates the location of the point electrode. 